\title{
O EFEITO DE DIFERENTES ROTAS DE PROCESSAMENTO ASSOCIADAS A ENGENHARIA DE CONTORNO DE GRÃO E MELHORIA DAS PROPRIEDADES MECÂNICAS ${ }^{*}$
}

\author{
Melânea Almeida Ramalho Medeiros ${ }^{1}$ \\ Clarissa Hadad de Melo ${ }^{2}$ \\ Alessandra Vieira Guimarães ${ }^{3}$ \\ Matheus Campolina Mendes ${ }^{4}$ \\ Leonardo Sales Araújo ${ }^{5}$
}

\section{Resumo}

A liga de níquel 718 encontra aplicações variadas nas indústrias aeronáutica, aeroespacial, óleo e gás e nuclear em função da excelente combinação entre resistência e ductilidade. As diferentes formas de processamento utilizadas para sua elaboração tendem a unir processos de deformação com temperatura, a fim de melhorar as propriedades mecânicas do material. Sendo assim, o presente trabalho tem como objetivo a caracterização e investigação do efeito de rotas de processamento termomecânicos distintas, avaliando a repercussão na fração de contornos especiais, dos carbetos $(\mathrm{Nb}, \mathrm{Ti}) \mathrm{C}$ e nas propriedades mecânicas do material endurecido por precipitação.

Palavras-chave: Liga 718; Engenharia de contorno de grão; Tratamento termomecânico; Carbetos.

\section{EFFECT OF DIFFERENT PROCESSING ROUTES ASSOCIATED WITH GRAIN BOUNDARY ENGINEERING AND IMPROVEMENT OF MECHANICAL PROPERTIES}

\section{Abstract}

The nickel alloy 718 are the most varied applications in the aerospace, oil and gas and nuclear industries in excellent combination of function between resistance and ductility. The different forms of mechanical process used for their preparation usually combine deformation processes and temperature in order to improve the mechanical properties of the material. Thus, this study aims to characterize and investigate the effect of different thermomechanical process routes, evaluating the impact on the special boundary fraction, the carbides $(\mathrm{Nb}, \mathrm{Ti}) \mathrm{C}$ and mechanical properties of a precipitation hardenend material.

Keywords: Alloy 718; Grain boundary engineering; Thermomechanical; Carbides.

1 Engenheira de Materiais, Mestranda em Engenharia Metalúrgica e de Materiais, Programa de Engenharia Metalúrgica e de Materiais, COPPE, Universidade Federal do Rio de Janeiro, Rio de Janeiro, RJ, Brasil.

2 Engenheira Metalúrgica, Mestranda em Engenharia Metalúrgica e de Materiais, Programa de Engenharia Metalúrgica e de Materiais, COPPE, Universidade Federal do Rio de Janeiro, Rio de Janeiro, RJ, Brasil.

3 Estudante de Engenharia de Materiais, Universidade Federal Rural do Rio de Janeiro, Rio de Janeiro, RJ, Brasil

4 Engenheiro de Materiais, MSc, Doutorando em Engenharia Metalúrgica e de Materiais, Programa de Engenharia Metalúrgica e de Materiais, COPPE, Universidade Federal do Rio de Janeiro, Rio de Janeiro, RJ, Brasil.

5 Engenheiro Metalúrgico, DSc, Professor, Programa de Engenharia Metalúrgica e de Materiais, COPPE, Universidade Federal do Rio de Janeiro, Rio de Janeiro, RJ, Brasil. 


\section{INTRODUÇÃO}

As ligas à base de níquel são especialmente desenvolvidas para atuarem sob condição de alta resistência mecânica a elevadas temperaturas. Em particular, a liga de níquel 718 apresenta um conjunto de propriedades excelente, com alta resistência e tenacidade desde temperaturas criogênicas de $-250^{\circ} \mathrm{C}$ até temperaturas mais elevadas de $650^{\circ} \mathrm{C}$, além de apresentar resistência a ambientes severos como equipamentos das indústrias petroquímica, termoelétrica, aeroespacial e nuclear [1,2]. Suas excelentes propriedades advêm do controle de sua microestrutura. A alta resistência mecânica da liga é induzida primordialmente pela precipitação das fases endurecedoras e metaestáveis $\gamma^{\prime}\left(\mathrm{Ni}_{3}(\mathrm{Al}, \mathrm{Ti})\right.$ ) e $\gamma^{\prime \prime}$ $(\mathrm{Ni} 3 \mathrm{Nb})$, sendo a última mais efetiva, distribuindo-se uniformemente na matriz austenítica $\gamma$. Além destas, as principais fases usualmente observadas são a fase $\delta\left(\mathrm{Ni}_{3} \mathrm{Nb}\right)$ e os carbetos $(\mathrm{Nb}, \mathrm{Ti}) \mathrm{C}[3,4]$.

A laminação a quente é um processo de deformação primária bastante eficiente, em que a variação de temperatura durante o procedimento pode intervir na distribuição de tensões e deformações alterando a microestrutura do material. A caracterização microestrutural e o comportamento recristalizado da liga 718 deformada a quente têm sido estudado para se obter um melhor controle do processamento termomecânico e desempenho do material [5].

Outra forma de processamento para melhorar as propriedades mecânicas da liga 718 é através da distribuição característica dos contornos de grão, conhecida como engenharia de contorno de grão. Essa técnica sugere o uso de tratamento termomecânico através de deformação em passe único ou múltiplos passes e recozimento subsequente, a fim de aumentar a fração de contornos especiais e melhorar suas propriedades. O parâmetro $\Sigma$ foi definido para descrever a densidade do modelo de sítios de coincidência (CSL), sendo os contornos de baixo- $\Sigma$ frequentemente chamados de "contornos especiais", devido a uma menor segregação de impureza, maior resistência à oxidação e nucleação e propagação de trincas [6]. Os CSLs gerados pelo processamento da engenharia de contorno de grão para ligas com baixa energia de falha de empilhamento são $\Sigma 3^{n}(\Sigma 3, \Sigma 9$ e $\Sigma 27)$, com maclas de recozimento coerentes e incoerentes, sendo o $\Sigma 3$ um contorno de grande contribuição para melhoria das propriedades devido a sua baixa energia $[6,7]$.

Alguns autores [5,8] compararam o efeito de diferentes parâmetros de processamento termomecânico, na laminação a frio associada a engenharia de contorno grão da liga 718.

Desta forma, o objetivo desse trabalho é investigar o efeito de duas diferentes rotas de processamento termomecânico, laminação a quente e engenharia de contorno de grão, a fim de melhorar as propriedades mecânicas do material.

\section{MATERIAIS E MÉTODOS}

Uma chapa da liga 718 de $10 \mathrm{~mm}$ de espessura e composição apresentada na Tabela 1 , foi processada termomecanicamente utilizando duas rotas diferentes. A primeira rota, foi nomeada por LQ e a segunda por ECG. Na rota LQ, a amostra foi submetida a homogeneização por 20 minutos a $1050^{\circ} \mathrm{C}$ e em seguida a quatro etapas de laminação a quente, com $20 \%$ de deformação por passe e subsequente solubilização a $1050^{\circ} \mathrm{C}$ por $1 \mathrm{~h}$, chegando a uma espessura final de $3 \mathrm{~mm}$. A rota ECG foi escolhida de acordo com o estudo realizado por Krupp et al. [8] onde foi 
executado quatro etapas de laminação a frio, com $20 \%$ de deformação seguido de solubilização a $1050^{\circ} \mathrm{C}$ por $1 \mathrm{~h}$, chegando a uma espessura final de $4 \mathrm{~mm}$. As amostras resultantes das rotas LQ e ECG foram envelhecidas a $760^{\circ} \mathrm{C}+4 \mathrm{~h} 45^{\prime}+$ resfriamento $38^{\circ} \mathrm{C} / \mathrm{h}+650^{\circ} \mathrm{C} / 1 \mathrm{~h} 15^{\prime}$

Tabela 1. Composição química da chapa utilizada.

\begin{tabular}{lccccccc}
\hline Elemento & Ni & Cr & Fe & Nb & Mo & Al & Ti \\
\hline \% Peso & 53,7 & 18,1 & 18,1 & 5,15 & 3,03 & 0,47 & 1,01 \\
Elemento & Co & Mn & Si & C & S & P & Cu \\
\hline \% Peso & 0,13 & 0,24 & 0,09 & 0,053 & 0,002 & 0,005 & 0,006 \\
\hline
\end{tabular}

Para a observação da microestrutura em microscópio eletrônico de varredura, as amostras foram lixadas, polidas e submetidas à limpeza em ultra-som. Após a preparação metalográfica, as amostras foram polidas em sílica coloidal por 25 minutos para posterior análise de EBSD. Para essa análise, o step size utilizado foi de $2 \mu \mathrm{m}$. Essa análise além de caracterizar os contornos de grão e maclas, determina a fração e distribuição de contornos de baixa energia $\left(\Sigma 3^{n}\right)$.

Nas imagens obtidas por microscopia eletrônica de varredura, em modo retroespalhado, foram definidos campos aleatórios com aumento de 200x, de forma a evitar um viés na análise. Por binarização e análise do histograma, as imagens foram avaliadas utilizando o software Image J, determinando a fração e distribuição dos carbetos de nióbio e tamanho médio de partículas.

As propriedades mecânicas foram analisadas por ensaios de tração a uma taxa de deformação de $10^{-4} \mathrm{~s}^{-1}$ até a ruptura dos corpos de prova. A resistência à ruptura, limite de escoamento, e valores de alongamento foram obtidos pela curva tensãodeformação

\section{RESULTADOS E DISCUSSÃO}

A Tabela 2 apresenta a soma da fração dos contornos de $\Sigma 3, \Sigma 9, \Sigma 27$ e o tamanho de grão calculado após o processamento das duas rotas em estudo. Observa-se que tanto para a rota LQ quanto para a rota ECG houve um aumento de $42 \%$ na fração de contornos especiais e $60 \%$ no tamanho de grão, em relação a amostra como recebida.

A rota ECG obteve resultados similares com o estudo realizado por Krupp et al. [8], onde os autores também utilizaram quatro ciclos de processamento combinando laminação a frio com grau de redução de $20 \%$ seguido de solubilização a $1050^{\circ} \mathrm{C} / 1 \mathrm{~h}$. Os autores obtiveram uma soma da fração de contornos de $20.9 \%$ para amostra recebida e $41 \%$ para a amostra após o processamento, o que torna um resultado coerente com o obtido na rota ECG referenciada nesse estudo. Lee et al. [10] atribuem o crescimento do grão ao aumento da maclas $(\Sigma 3)$ e essa relação se torna mais evidente quando observamos a rota LQ. O tamanho de grão da amostra ECG foi ligeiramente menor que na amostra $L Q$. 
Tabela 2. Fração de contornos especiais e tamanho de grão para as amostras das rotas A e B e como recebida.

\begin{tabular}{lccccc}
\hline AMOSTRA & $\mathbf{\Sigma 3}$ & $\boldsymbol{\Sigma 9}$ & $\boldsymbol{\Sigma} \mathbf{2 7}$ & $\mathbf{\Sigma 3 +} \mathbf{\Sigma 9 +} \boldsymbol{\Sigma} \mathbf{2 7}$ & $\begin{array}{c}\text { Tamanho } \\
\text { de grão } \\
((\boldsymbol{\mu m})\end{array}$ \\
\hline Como recebida & 26,0 & 0,5 & 0,01 & 26,51 & 14 \\
Rota LQ & 45,5 & 0,3 & 0,2 & 46,0 & 35 \\
\hline Rota ECG & 44,0 & 0,4 & 0,1 & 44,5 & 32 \\
\hline
\end{tabular}

A microestrutura após as etapas de processamento termomecânico foi avaliada por microscopia eletrônica de varredura. A microestrutura consistiu de grãos equiaxiais com a presença de partículas de $(\mathrm{Nb}, \mathrm{Ti}) \mathrm{C}$ na forma de agrupamentos alinhados à direção de laminação. Tais carbetos do tipo MC são oriundos ainda da solidificação da liga, na forma de carbetos primários, os quais são fragmentados durante as etapas subsequentes de processamento termomecânico. A Figura 1 mostra a microestrutura. A fragmentação destes carbetos foi determinada por análise de imagem e sua fração é próxima aquela estimada termodinamicamente em [9]. Os resultados obtidos mostram que na rota ECG a amostra apresenta menor fração de área e menor tamanho de partícula que a amostra da rota $L Q$, na condição solubilizada. Isso se deve a fragmentação das partículas de carbetos na rota ECG, enquanto na rota $L Q$ reduz a fragmentação devido a melhor acomodação das mesmas na matriz. Além disso, nota-se que no caso da rota ECG houve uma menor distribuição das partículas maiores na matriz, deixando-as menos dispersas. Os resultados de tamanhos médios de partículas e fração de área são apresentados na Tabela 3.
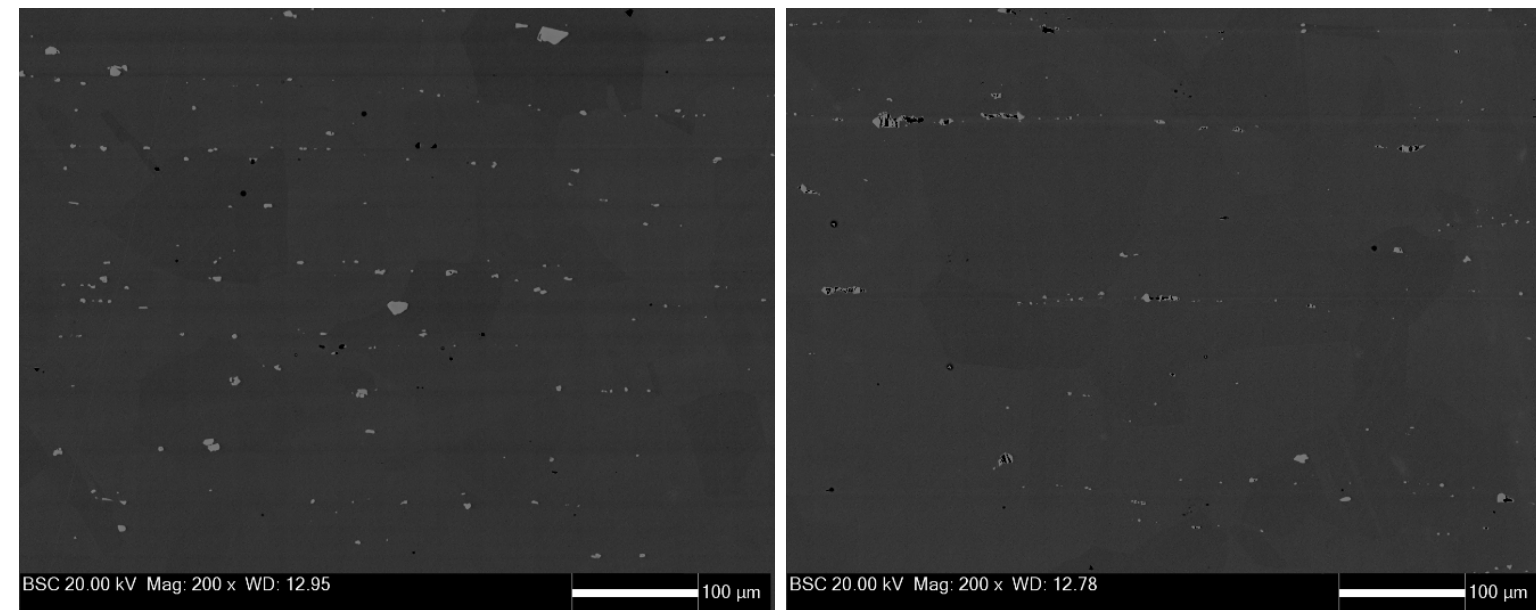

Figura 1. Imagens de MEV. (a) Amostra obtida pela rota LQ; (b) Amostra obtida pela rota ECG. 
Tabela 3. Tamanho de partícula e fração de área para as rotas LQ e ECG [9]

\begin{tabular}{lcc}
\hline AMOSTRA & $\begin{array}{c}\text { FRAÇÃO DE ÁREA } \\
\text { DE }(\mathbf{N b}, \mathrm{Ti}) \mathbf{C}\end{array}$ & $\begin{array}{c}\text { TAMANHO MÉDIO DE } \\
\text { PARTÍCULAS }(\mu \mathrm{m})\end{array}$ \\
\hline Rota LQ & $0,56 \pm 0,06$ & $12,88 \pm 0,76$ \\
Rota ECG & $0,47 \pm 0,03$ & $9,57 \pm 1,14$ \\
\hline
\end{tabular}

Os resultados do ensaio de tração e a comparação destes valores com o tamanho médio de grão, para cada condição de tratamento térmico do material, são apresentados na Tabela 4. Comparando as rotas LQ e ECG entre si, a amostra solubilizada apresentou maior limite de escoamento para a amostra ECG, sem, contudo, impactar no limite de resistência e na ductilidade, a qual foi similar a amostra LQ. O maior valor do limite de escoamento para a amostra ECG, sugere uma alteração microestrutural induzida pelo processamento na amostra. Dado que as frações de contornos especiais ficaram próximas, assim como o tamanho de grão, acredita-se que o menor tamanho de partículas de ( $\mathrm{Nb}, \mathrm{Ti}) \mathrm{C}$ possa ter contribuído para o endurecimento da amostra ECG em comparação a LQ. Dada a grande ductilidade da matriz no estado solubilizado, tal efeito não foi significante no alongamento.

Tabela 4. Resultados do ensaio de tração.

\begin{tabular}{|l|l|c|c|c|}
\hline \multirow{2}{*}{ AMOSTRA } & $\boldsymbol{\sigma}_{\mathrm{LE}}(\mathrm{Mpa})$ & $\boldsymbol{\sigma}_{\mathrm{LR}}$ (Mpa) & Al (\%) \\
\hline \multirow{2}{*}{ Solubilizada } & Rota LQ & $233,5 \pm 22,6$ & $761,6 \pm 0,9$ & $78,5 \pm 2,1$ \\
\cline { 2 - 5 } & Rota ECG & $304,5 \pm 4,26$ & $750,46 \pm 1,63$ & $78,5 \pm 0,71$ \\
\hline \multirow{2}{*}{ Envelhecida } & Rota LQ & $1147,2 \pm 2,1$ & $1290,9 \pm 16,7$ & $21,2 \pm 1,1$ \\
\cline { 2 - 5 } & Rota ECG & $1057,4 \pm 1,2$ & $1293,3 \pm 9,3$ & $24,4 \pm 0,6$ \\
\hline
\end{tabular}

Já para as amostras envelhecidas, o limite de escoamento da amostra ECG foi ligeiramente maior, mas sua diferença em relação a amostra LQ foi muito reduzida dada a preponderância da precipitação como mecanismo de endurecimento. Contudo, para as amostras nessa condição, foi notada uma maior ductilidade, manifestada em um maior alongamento, para a amostra ECG, quando comparada a amostra LQ. Para a observação dessas amostras, foi realizada a fractografia dos corpos de provas, apresentados na Figura 2. Nota-se que, para a amostra ECG, as microcavidades apresentam maior profundidade. Além disso, observa-se que maiores microcavidades são concentradas em certas regiões da superfície de fratura, enquanto no caso da LQ, são mais distribuídas, apesar de seu maior tamanho. Isso pode ser corroborado pelo aspecto observado nas amostras ECG, como uma menor dispersão das partículas de $(\mathrm{Nb}, \mathrm{Ti}) \mathrm{C}$, apesar de seu menor tamanho médio. O estudo de Goodwin e colaboradores [11] mostrou que a maior ductilidade do material pode ser associada por um maior espaço interpartículas, o qual permite um maior crescimento do vazio induzido pela deformação, antes que tal crescimento cause a fratura. No caso da amostra ECG, apesar do menor tamanho médio das partículas, estas se encontram mais concentradas em determinadas regiões, em comparação às amostras $L Q$, onde o $(\mathrm{Nb}, \mathrm{Ti}) \mathrm{C}$ se dispersou mais ao longo da microestrutura. Tal fato não foi sensível nas amostras solubilizadas, dada a 
grande ductilidade da matriz nesse estado. Contudo, nas amostras envelhecidas, a natural restrição a deformação plástica da matriz imposta pelo endurecimento provocado pela precipitação de $\gamma^{\prime}$ e $\gamma^{\prime \prime}$ fez com que esse efeito fosse significativo a ductilidade.
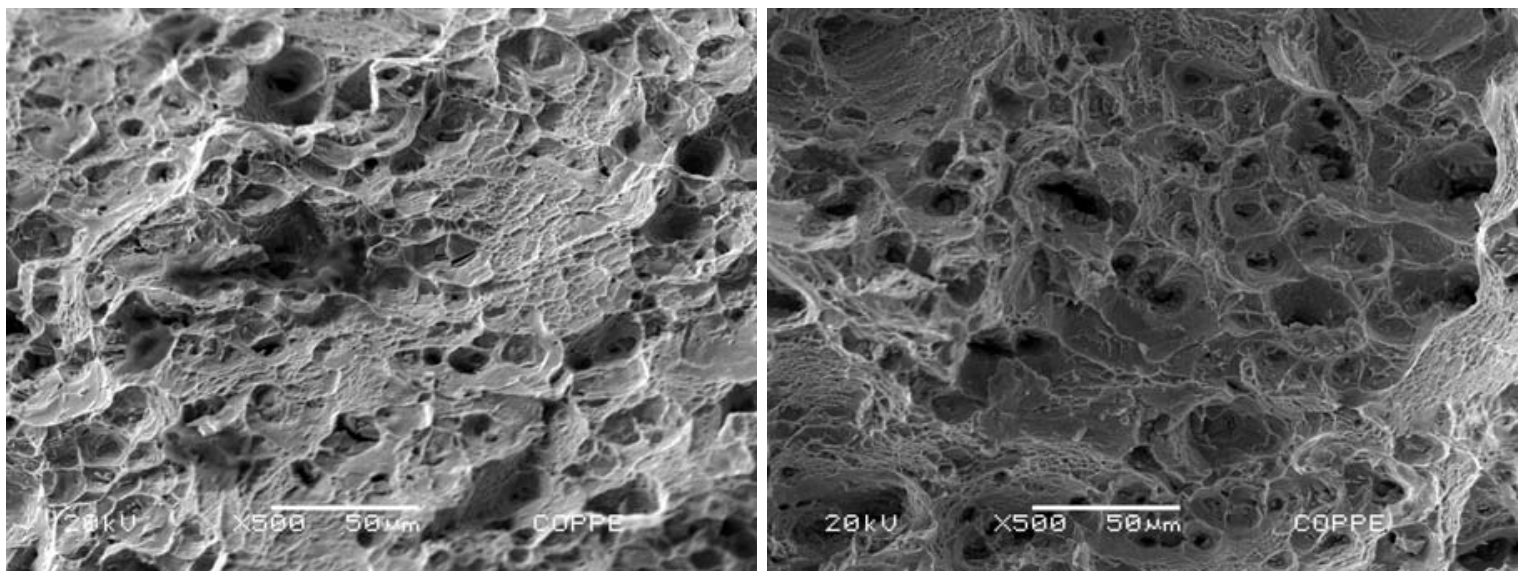

Figura 2. Imagens de MEV. (a) superfície de fratura da amostra LQ; (b) superfície de fratura da amostra ECG.

\section{CONCLUSÃO}

Baseado no presente trabalho, pode-se concluir que tanto as rota de processamento por laminação a quente (LQ) quanto a rota de engenharia de contorno de grão (ECG) foram efetivas no aumento da fração de contornos especiais em relação ao material como recebido. Contudo, a rota ECG, dado o seu caráter de deformação a frio, promoveu maior fragmentação de partículas dos carbetos primários $(\mathrm{Nb}, \mathrm{Ti}) \mathrm{C}$. Essa maior fragmentação induziu endurecimento da amostra solubilizada ECG, em comparação a LQ. Portanto, após o tratamento térmico de precipitação, o endurecimento promovido por $\gamma^{\prime}$ e $\gamma^{\prime \prime}$ foi amplamente preponderante, reduzindo significativamente a diferença de limite de escoamento entre as amostras ECG e LQ. Apesar do menor tamanho de partículas da amostra ECG, a sua maior concentração em determinadas regiões promoveu uma ligeira alteração da morfologia de fratura dos corpos de provas e maior ductilidade. O maior espaçamento entre os agrupamentos de partículas pode ter contribuído para o maior crescimento dos vazios induzidos pela deformação, atrasando o processo de fratura.

\section{Agradecimentos}

Os autores gostariam de agradecer o apoio financeiro do $\mathrm{CNPq}$, Capes e ao Laboratório de Materiais do CEFET/RJ.

\section{REFERÊNCIAS}

1 Jewet RP, Halchak JA. The use of alloy 718 in space shuttle main engine.Insuperalloys 718, 625 and various derivatives, 1991.

2 American petroleum institute, api6a718 - specification of nickel-base alloy 718 (uns n07718) for oil and gas drilling and production equipment, american petroleum institute, 2004.

3 Donachie M, Donachie S. A Technical Guide, Materials Park, OH: ASM International, 2002. 
4 Araujo, LS. processamento e caracterização de superliga de níquel 718 para aplicações nucleares, tese de doutorado, Programa de Pós-graduação em EngenhariaMetalúrgica e de Materiais, COPPE/UFRJ, Rio de Janeiro, 2012.

5 Wang Y, Shao WZ, Zhen L, Zhang XM. Microstructure evolution during dynamic recrystallization hot deformed of superalloy 718 . Materials Science and Engineering. 2008; A 486: 321-332.

6 TanL, AllenTR,BusbyJT.Grain boundary engineering for structure materials of nuclear reactors. Journal of nuclear materials.2013; 441(1-3): 661-666.

7 Tan L, Sridharan, K, Allen TR. Effect of thermomechanical processing on grain boundary character distribution of a ni-based superalloy. Journal of nuclear materials. 2007; 371 (1): 171-175.

8 Krupp U, Kane W, Liu X, Deuebner O, Laird C, Mcmahon C. The effect of grainboundary-engineering-type processing on oxygen-induced cracking if in718. Materials Science and Engineering.2003; A 319: 213-217.

9 Guimarães AV, Araujo LS. Análise da morfologia de carbetos (Nb, Ti)C da superligas de níquel 718 após processo termomecânico, $15^{\circ}$ ENEMET - Encontro Nacional de Estudantes de Engenharia Metalúrgica, de Materiais e de Minas. 2015;

10 S.-L. Lee and N. Richards, The effect of single-step low strain and annealing of nickel on grain boundary character, Materials Science and Engineering. 2005; 390(1-2): 8187.

11 Goodwin SJ, Noble FW, EYRE BL. Inclusion nucleated ductile fracture stainless steel. Acta metal. 1989; 37 (5); 1389-1398. 